Revista Arbitrada Interdisciplinaria KOINONIA

Año VI. Vol VI. N4. Edición Especial: Educación III. 2021

Hecho el depósito de Ley: FA2016000010 ISSN: 2542-3088

FUNDACIÓN KOINONIA (F.K). Santa Ana de Coro. Venezuela.

Verónica Marisol Tene-Seas; Sandra Elizabeth Mena-Clerque

http://dx.doi.org/10.35381/r.k.v6i4.1498

\title{
Gamificación como estrategia para la enseñanza - aprendizaje de Emprendimiento y Gestión
}

Gamification as a strategy for the teaching - learning of Entrepreneurship and Management

\author{
Verónica Marisol Tene-Seas \\ veronica.tene.70@est.ucacue.edu.ec \\ Universidad Católica de Cuenca, Yacuambi \\ Ecuador \\ https://orcid.org/0000-0002-6263-9286 \\ Sandra Elizabeth Mena-Clerque \\ sandramena@ucacue.edu.ec \\ Universidad Católica de Cuenca, Cuenca \\ Ecuador \\ https://orcid.org/0000-0002-9186-2161
}

Recepción: 30 de agosto 2021

Revisado: 20 de septiembre 2021

Aprobación: 15 de noviembre 2021

Publicación: 01 de diciembre 2021 
Revista Arbitrada Interdisciplinaria KOINONIA

Año VI. Vol VI. N4. Edición Especial: Educación III. 2021

Hecho el depósito de Ley: FA2016000010

ISSN: 2542-3088

FUNDACIÓN KOINONIA (F.K). Santa Ana de Coro. Venezuela.

Verónica Marisol Tene-Seas; Sandra Elizabeth Mena-Clerque

\title{
RESUMEN
}

La gamificación es una herramienta importante en la educación, ya que nos ayuda como un escenario digital, por lo que podemos trabajar con diferente herramientas y aplicaciones móviles, que son netamente diseñados y utilizados para realizar un aprendizaje interactivo y significativo de acuerdo a las plataformas utilizadas, siendo de estos algunos gratuitos. El objetivo de la investigación es analizar de que forma la gamificación puede emplearse para la enseñanza - aprendizaje de Emprendimiento y Gestión en primer año bachillerato intensivo. De metodología descriptiva. El 96,3\% de los resultados obtenidos en la encuesta realizada a los alumnos se encontraban satisfechos, por cuanto creen que es importante y necesario que en esta actualidad se pueda identificar y conocer cómo se llevaría a cabo el uso de la gamificación y podría ser empleado el mismo mediante la educación. A los alumnos les gustaría trabajar con los diferentes elementos de la gamificación, ya que en cada elemento se podría utilizar distintas formas para poder incentivar y de tal manera realizar las actividades que se tengan planificadas.

Descriptores: Juego educativo; material didáctico; programa informático didáctico. (Palabras tomadas del Tesauro UNESCO).

\begin{abstract}
Gamification is an important tool in education, since it helps us as a digital setting, so we can work with different tools and mobile applications, which are clearly designed and used to carry out interactive and meaningful learning according to the platforms used., some of which are free. The objective of the research is to analyze how gamification can be used for the teaching - learning of Entrepreneurship and Management in the first year of intensive high school. Descriptive methodology. 96.3\% of the results obtained in the survey carried out with the students were satisfied, because they believe that it is important and necessary that at this time it is possible to identify and know how the use of gamification would be carried out and it could be employed himself through education. The students would like to work with the different elements of gamification, since in each element different forms could be used to be able to incentivize and in such a way carry out the activities that are planned.
\end{abstract}

Descriptors: Educational games; teaching materials; educational software. (Words taken from the UNESCO Thesaurus). 


\section{INTRODUCCIÓN}

Según (Méndez-Escobar, 2020), por la aparición de la pandemia del COVID-19 nos vimos afectados en todos los ámbitos a nivel mundial, especialmente en el ámbito educativo por lo que nos encontramos forzados a cerrar todas las instituciones educativas a nivel mundial para de esa manera poder evitar contagios, por otro lado, tuvimos que trabajar de una manera diferente, por lo que se optó trabajar en las clases virtuales utilizando plataformas mediante el uso tecnológico.

Por consiguiente, nos tocó adoptar diferentes técnicas de aprendizaje para poder trabajar con los alumnos mediante las clases en línea, se puede señalar que la educación en el Cantón Yacuambi se vio afectado directamente para todos los habitantes, por cuanto todos no poseen internet y equipos informáticos, por lo que a un grupo de alumnos se les complicaba ingresar y poder entender las clases, en pocas palabras no podrán tener un desenvolvimiento adecuado. De los descrito en el párrafo anterior creo conveniente estudiar este tema, ya que como docentes debemos buscar diferentes estrategias para trabajar con nuestros alumnos, de esa manera podamos analizar de qué forma la gamificación puede emplearse en la enseñanza - aprendizaje en Emprendimiento y Gestión con los alumnos de primero año bachillerato.

El presente trabajo se enfoca en como beneficiará el uso de los elementos y componentes de la gamificación mediante la estrategia metodológica en la enseñanza aprendizaje de emprendimiento y gestión de primer año de bachillerato, buscando que se desarrolle de una manera de fácil acceso a cada estudiante y a la vez ellos destacarse por ese medio.

Por el estado de emergencia el Ministerio de Educación opto por realizar el Plan Educativo COVID-19 en el que se encuentra orientado a garantizar la educación a nivel nacional con la intención de entregar todas las herramientas pedagógicas y metodológicas con el fin de trabajar mediante las plataformas virtuales a la vez realizar sus respectivas adaptaciones curriculares de acuerdo a las necesidades que se vayan presentando de eso modo poder dar seguimiento a las clases virtuales efectuadas en 
periodo de pandemia de esa manera evitar que hayas alumnos rezagados en este periodo electivo (Ortiz-Colón et al. 2018).

Por parte de nuestra institución optamos por mantenernos en las clases virtuales mediante la plataforma Microsoft Teams con el fin de evitar la propagación del virus, de tal manera que trabajamos con el Plan Educativo COVID-19 que fue emitido por el Ministerio de Educación. Por esta razón considero que este trabajo es muy importante para ayudar con las habilidades, destrezas, conocimientos, desenvolvimiento y desempeño de los alumnos en las clases en virtuales y presencial basándonos en el uso de la gamificación, en otras palabras, de esta forma tomar las medidas necesarias para mejorar las estrategias metodológicas en la enseñanza - aprendizaje de emprendimiento y gestión en los mismo y de esta manera poder cumplir con los objetivos plateados.

Además, sería de gran beneficio para los padres de familia, alumnos y docentes en el Cantón Yacuambi, ya que por la pandemia se vio afectada la educación, por ende, el uso de la gamificación mediante la dinámica, mecánica y componentes del juego que nos brindan ventajes al momento de diseñar la estrategia metodológica para trabajar en las clases tanto en virtuales como de manera presencial, con el fin de obtener participación activa y buscar el interés por parte de los alumnos y de tal forma obtener excelentes resultados académicos.

\section{Referencial teórico}

\section{Enseñanza - Aprendizaje}

El concepto basico de enseñanza se puede descirbir en realizar las distintas actividades que le revelen a los estudiantes un acontencimiento histórico, teórico y particular, sin embargo, los procesos nos llevan a emplear diferentes modelos de aprendizaje todos relaciones a medir el nivel de conocimientos significativos de los estudiantes. (BurgosBonozo, 2019) En el proceso de enseñanza-aprendizaje se utilizan estrategias para que el alumno interprete y aprenda a través de distintas herramientas diseñadas por el 
docente falicitador que le ayuda a desarrollar sus desrezas y habilidades (TorresToukoumidis et al. 2018).

El constructivismo como aprendizaje señala que el estudiante crea su propio conocimiento con la ayuda de los conocimientos que ya posee, por lo que la materia de Emprendimiento y Gestió se basa en el constructivismo por lo que se aspira a que el estudiante este preparado para ser una persona dinamica, activa y que pueda crear su propio conocimiento apoyandose de las nuevas tecnologias y a la vez mantener una comunicación asertiva con el docente con el fin de acceder a un aprendizaje significativo. La materia de Emprendimiento busca la manera de ser una aprendizaje significativo con el fin de poder poner en practica todos los conocimientos adquiridos y a la vez desarrollar sua habilidades y destrezas mediante una estrategia de enseñanza - aprendizaje. (Yánez-Analuisa, 2020)

\section{La Gamificación}

Pretende trabajar de una manera significativa para la educación buscando, estimulando y aumentando sus conocimientos y habilidades con el propósito de obtener mayor compromiso académico por parte de cada uno de los alumnos de esta manera proceder a motivarlos con recompensas a través de las dinámicas, mecánicas y componentes del juego con sus respectivas adaptaciones de acuerdo a las diferentes áreas de estudios de esa manera obtener un mejor desenvolvimiento y buenos resultados académicos. (Liberio-Ambuisaca, 2019).

\section{Tipos de Gamificación}

Ante todo, (García et al. 2020) en España definen dos tipos de gamificación, la primera ayuda al trabajo directamente con el contenido dependiendo del área de estudio y el segundo se encuentra orientado con la organización y estructura de la clase. Por ende, evidentemente para proceder a gamificar se requiera de algunos elementos como lo son los siguientes: 
a. Determinar las reglas del juego.

b. Indicar que componentes, mecánicas y dinámicas se utilizaran durante las actividades.

c. Concretar el contenido y características que sean coherentes para los estudiantes.

d. Especificar el propósito del juego desde un inicio.

e. Enunciar las regalas de forma clara, precisa y concisa.

f. Crear grupos de forma equitativa.

g. Establecer la recompensa que obtendrán conforme vayan avanzando y al finalizar el juego.

\section{Componentes de la Gamificación}

Consiste en dinámicas, mecánicas y componentes del juego siendo un factor importante al momento de ejecutar la gamificación los cuales se podrán desarrollar conjuntamente con los elementos antes ya mencionados y de esa manera relacionar con los contenidos educativos de acuerdo al área de estudio (Gaspar et al. 2016).

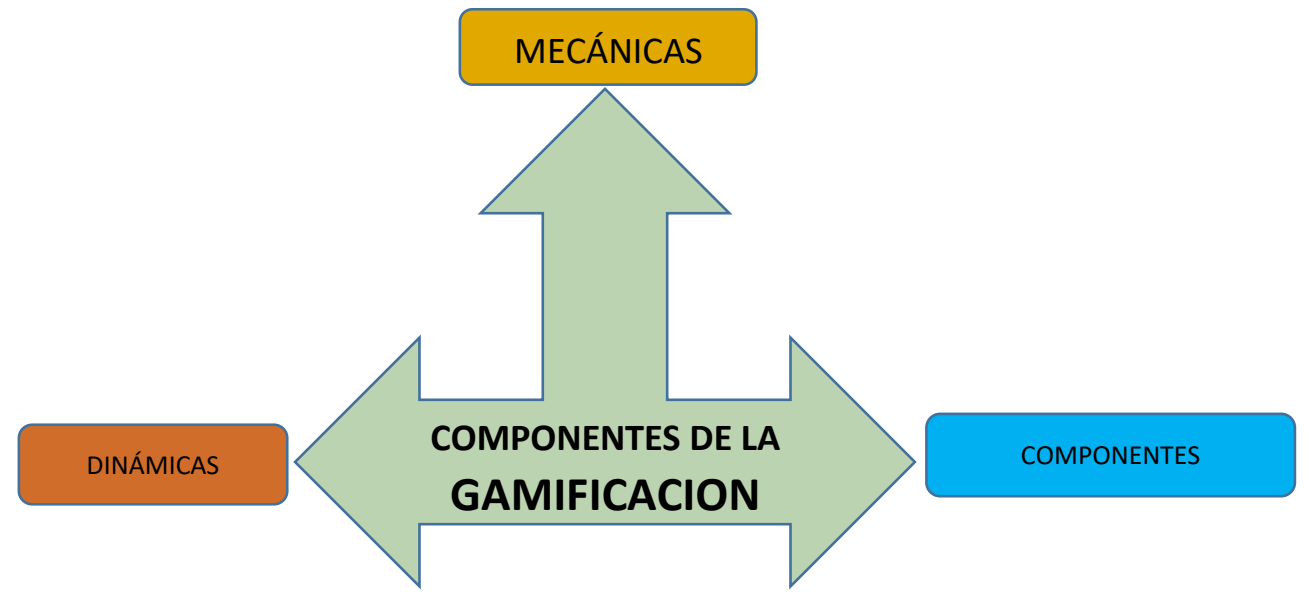

Figura 1. Elementos de la gamificación.

Elaboración: Los autores. 
En primero lugar las dinámicas de juego son aquellas que permiten motivar a los estudiantes al momento de llevar a cabo alguna actividad, teniendo en cuenta que se busca la forma de recompensar la cooperación, compañerismo y el desenvolvimiento que presenten en las actividades ejecutadas. A continuación, se observa algunos ejemplos que podemos emplear:

a. Recompensa: es la que se lleva el o los participantes que logran completar con todos los retos encomendados.

b. Estatus: son los niveles que obtiene cada grupo o participante de acuerdo a los retos logrados, de esa manera motivar la participación activa en los diferentes retos que se propongan trabajar.

c. Logro: es el que se obtiene por superar las diferentes adversidades presentadas en cada uno de los retos.

d. Expresión: es la que permite identificar las personalidades, gustos, características y debilidades por medio de un avatar.

e. Competición: tiene como objeto de que los alumnos realicen una competencia sana, sea de manera individual o grupal, siempre con respeto hacia sus compañeros de esa forma poder esforzarse e intentar sobresalir victoriosos en los retos.

En segundo lugar, las mecánicas de los juegos se tratan de establecer las reglas y retos que se presentaran en el transcurso que se lleve a cabo el juego. Siendo las principales las siguientes:

a. Puntos: se llevan a cabo en una tabla mediante un conteo que se realiza conforme avanzan y obtienen retos victoriosos por participante o grupo.

b. Niveles: varían de acuerdo a las dificultades que resuelvan los alumnos según los conocimientos adquiridos variara el nivel de cada participante.

c. Premios: son establecidos por el docente a cargo, se diseñan de acuerdo al desenvolvimiento y conocimientos de los alumnos en la actividad. 
d. Clasificación: se dará en el orden de participación y finalización de los retos para permitir que los participantes se esfuercen en el aprendizaje según sus conocimientos.

e. Retos: se proponen al inicio de una actividad, sea de manera virtual o presencial y se organiza recompensas para cada participante o grupo al finalizar todos los retos propuestos.

En tercer lugar, los componentes del juego que se desarrollan como la base de los juegos que se llevarían a cabo, ya que al finalizarlos se pueden obtener las recompensas. En efecto los componentes más relevantes son:

a. Avatares: son creados por los participantes según su personalidad, se mejoran conforme los niveles ganados y así acceder a los diferentes accesorios que posean.

b. Combate: es el que se ejecuta entre dos participantes o en grupos con el fin de competir con la misma actividad.

c. Colección: son las diferentes recompensas o premios que obtienen los participantes o grupos conforme ganen los retos.

d. Insignias: son las representaciones que se les entrega a los alumnos conforme vayan avanzando todos y cada uno de los retos ya que en cada reto que tenga podrán obtener una insignia y al finalizar todos los retos recibirán su recompensa.

La gamificación es una herramienta que puede convertir el aprendizaje en una actividad inmersiva. (Ortiz-Colón et al. 2018), se puede decir que al momento de aprender será de una manera diferente, divertida y por lo que pueda disfrutar en lo que se va trabajando, de tal forma que se encuentra incentiva para finalizar la tarea encomendad. También por medio de la gamificación podemos obtener una calidad educativa ya que podemos encontrar diferentes formas de trabajar mediante redes o incluso en un salón de clase, siempre y cuando buscando la forma de incentivar a los alumnos y motivarlos a una 
competencia sana y su a vez que pongan más atención en los temas estudiados, de esa manera podríamos obtener excelentes resultados académicos (Bernal-Suarez et al. 2015).

Además, la gamificación es una herramienta importante en la educación, ya que nos ayuda como un escenario digital, por lo que podemos trabajar con diferente herramientas y aplicaciones móviles, que son netamente diseñados y utilizados para realizar un aprendizaje interactivo y significativo de acuerdo a las plataformas utilizadas, siendo de estos algunos gratuitos (Torres-Toukoumidis et al., 2018).

\section{Aprendizaje basado en el juego}

En el podemos seguir las mecánicas de los juegos para poder resolver las actividades encomendadas, además no podríamos utilizar todos los elementos de la gamificación, sino irlos motivando a interactuar de una manera asertiva y dinámica por el simple hecho de participar en el juego. De tal forma que el juego sería más por diversión y tener nuevas experiencias educativas, concretando sus nuevos contenidos y conocimientos específicos según el juego a desarrollarse (Francés Monllor, 2019).

\section{Aprendizaje Game-like}

Mediante este aprendizaje se aplicara los premios de tal manera que podemos centrarnos más en el contenido que vamos a trabajar, por lo cual buscaremos las aplicaciones y herramientas que podamos utilizar de acuerdo a los contenidos a trabajar, con el fin de realizar las clases dinámicas, divertidas para poder llamar la atención de los alumnos y a la vez definir las reglas y premios que se usaran en los juegos que se llevaran a cabo (García Casaus et al. 2021). 
Revista Arbitrada Interdisciplinaria KOINONIA

Año VI. Vol VI. N4. Edición Especial: Educación III. 2021

Hecho el depósito de Ley: FA2016000010 ISSN: 2542-3088

FUNDACIÓN KOINONIA (F.K). Santa Ana de Coro. Venezuela.

Verónica Marisol Tene-Seas; Sandra Elizabeth Mena-Clerque

\section{METODOLOGÍA}

La presente investigación es tipo descriptiva con diseño no experimental y la metodología que su utiliza es cuantitativa con enfoque transversal por que se recolecto y analizó los datos (Erazo-Álvarez, 2021). La población fue de 27 alumnos de primero de BGU intensivo, con la finalidad de recopilar los datos para poder proceder al análisis correspondiente, por la cual se desarrolló la encuesta que se aplicó mediante instrumento tipo cuestionario el cual consta de 13 preguntas y se validó mediante el análisis de fiabilidad obteniendo como resultado estadístico el 0,910 de Alfa de Cronbach. (Carhuancho et al., 2019). Los datos obtenidos se procesaron mediante estadística descriptiva.

\section{RESULTADOS}

Según los resultados obtenidos en la investigación podemos identificar que tiene un paradigma positivista el cual se lo califica como cuantitativo de acuerdo al análisis pertinente, realizado a los resultados cuantitativos, determine que las variables analizadas mediante la encuesta que se efectúo tolas las respuestas nos indican que son variables paramétricas (Ricoy-Lorenzo, 2006). Mediante la aplicación de la encuesta realizada a los alumnos de primer año de bachillerato intensivo sección nocturna podemos evidenciar los siguientes resultados obtenidos de acuerdo a las siguientes tablas estadísticas. 


\section{Tabla 1}

Es necesario conocer la gamificación en la educación.

\begin{tabular}{llcccc}
\hline & Frecuencia & Porcentaje & $\begin{array}{c}\text { Porcentaje } \\
\text { válido }\end{array}$ & $\begin{array}{c}\text { Porcentaje } \\
\text { acumulado }\end{array}$ \\
\hline Válidos & Muy & 15 & 55,6 & 55,6 & 55,6 \\
& Importante & 11 & 40,7 & 40,7 & 96,3 \\
& Importante & 1 & 3,7 & 3,7 & 100,0 \\
& Regular & 27 & 100,0 & 100,0 & \\
\cline { 2 - 5 } & Total & & & \\
\hline
\end{tabular}

Fuente: Encuesta.

En la tabla 1 se observa que el $96,3 \%$ de los resultados obtenidos en la encuesta realizada a los alumnos se encontraban satisfechos, por cuanto creen que es importante y necesario que en esta actualidad se pueda identificar y conocer cómo se llevaría a cabo el uso de la gamificación y podría ser empleado el mismo mediante la educación.

\section{Tabla 2.}

Los juegos educativos son una buena estrategia de aprendizaje.

\begin{tabular}{llcccc}
\hline & Frecuencia & Porcentaje & $\begin{array}{c}\text { Porcentaje } \\
\text { válido }\end{array}$ & $\begin{array}{c}\text { Porcentaje } \\
\text { acumulado }\end{array}$ \\
\hline Válidos & Siempre & 25 & 92,6 & 92,6 & 92,6 \\
& Casi siempre & 1 & 3,7 & 3,7 & 96,3 \\
& En ocasiones & 1 & 3,7 & 3,7 & 100,0 \\
\cline { 2 - 5 } & Total & 27 & 100,0 & 100,0 & \\
\hline
\end{tabular}

Fuente: Encuesta. 
En la tabla 2 se observa que el $96,3 \%$ se hallan incentivados, ya que mediante los juegos educativos podrán ser motivados en el aprendizaje, sabiendo incrementar su atención y concentración de esa manera que los alumnos acepten sus errores para poder conseguir los mejores resultados al momento de ser empleados en la clase.

Tabla 3.

Frecuencia de utilización de la Gamificación en nuestras clases.

\begin{tabular}{llcccc}
\hline & & Frecuencia & Porcentaje & Porcentaje válido & $\begin{array}{c}\text { Porcentaje } \\
\text { acumulado }\end{array}$ \\
\hline Válidos & Siempre & 23 & 85,2 & 85,2 & 85,2 \\
& $\begin{array}{l}\text { Casi } \\
\text { siempre }\end{array}$ & 4 & 14,8 & 14,8 & 100,0 \\
\cline { 2 - 5 } & & & & \\
\hline
\end{tabular}

Fuente: Encuesta.

En la tabla 3 se encontró que el $85,2 \%$ cree que se debería utilizar siempre en uso de la gamificación en la clase, con el beneficio de sentirse motivados e incentivamos y a la vez buscando la forma de incrementar sus habilidades y conocimientos, de esa manera obtener sus excelentes resultados académicos, utilizando la gamificación por medio de la educación.

\section{PROPUESTA}

Desde mi punto de vista y basándome en los resultados obtenidos en la encuesta realiza a los alumnos, he considerado que se debe implementar la gamificación en las clases sea de manera virtual o presencial como una estrategia para la enseñanza-aprendizaje de Emprendimiento y Gestión con los alumnos bachillerato, por tal razón obtendríamos excelentes resultados académicos, ya que son estrategias innovadoras que utilizaríamos 
Revista Arbitrada Interdisciplinaria KOINONIA

Año VI. Vol VI. N4. Edición Especial: Educación III. 2021

Hecho el depósito de Ley: FA2016000010

ISSN: 2542-3088

FUNDACIÓN KOINONIA (F.K). Santa Ana de Coro. Venezuela.

Verónica Marisol Tene-Seas; Sandra Elizabeth Mena-Clerque

y seria del agrado de los alumnos, por lo que creo conveniente destacar los siguientes puntos detallados a continuación:

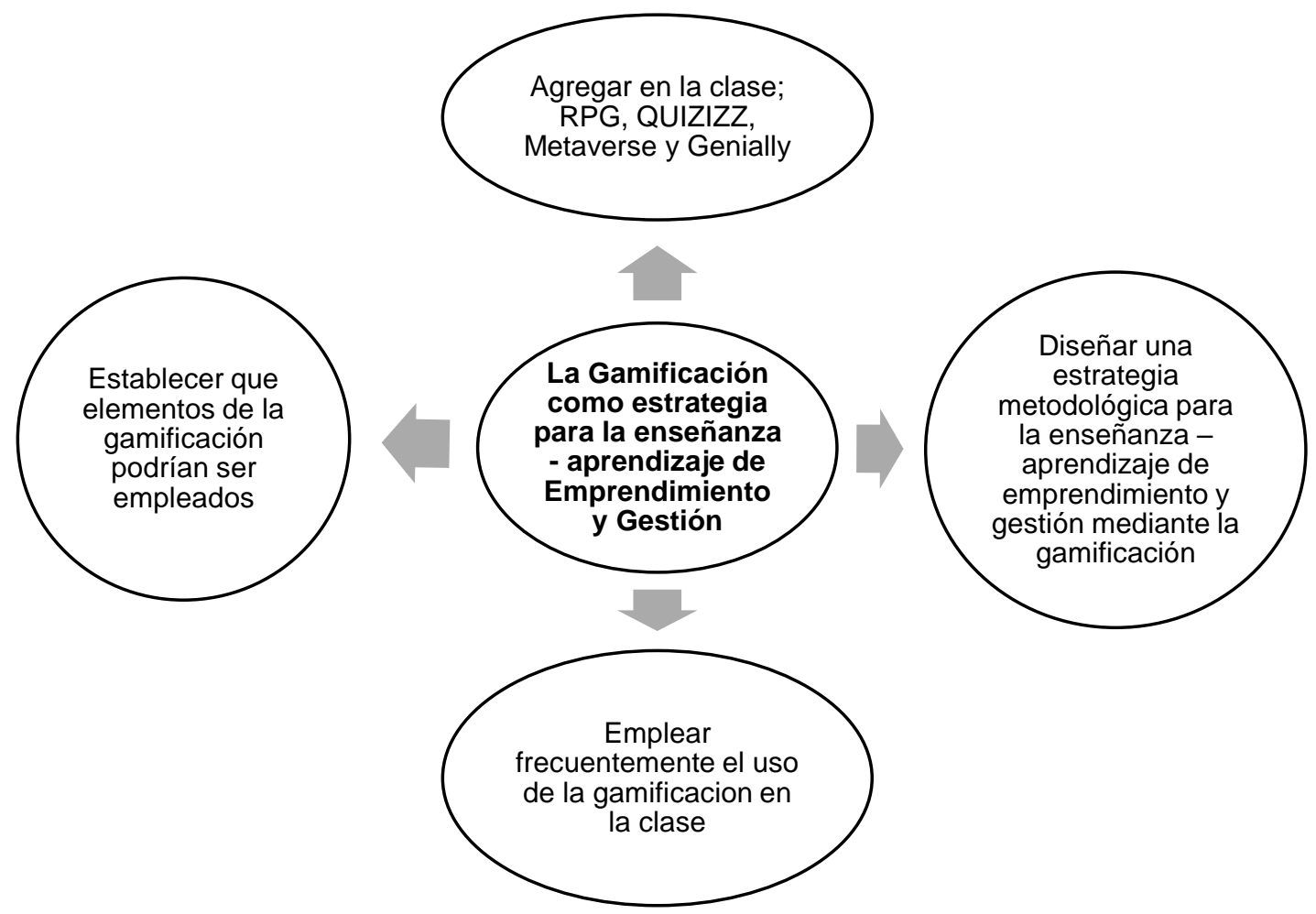

Figura 2. Gamificación como estrategia de enseñanza-aprendizaje de Emprendimiento y Gestión

Elaboración: Los autores.

\section{Agregar en la clase: RPG, QUIZIZZ, Metaverse y Genially.}

La gamificación empleada en la educación también se la realiza con las diferentes herramientas y aplicaciones utilizando dependiendo de los niveles educativos, por lo que he visto conveniente trabajar con las siguientes aplicaciones:
a. RPG
b. QUIZIZZ
c. Metaverse
d. Genially 
Por la cual al momento de ejecutarlas siempre describiríamos los componentes, mecánicas y dinámicas de los juegos que vamos a trabajar, de esa manera quedando claros con los componentes y elementos de la gamificación que utilizaremos mediante los juegos con contenidos interactivos, los cuales ayudaran a los alumnos a entender de mejor manera los temas a trabajar. Por medio de la gamificación podemos encontrar el uso de los elementos diseñados por videojuegos en el cual se traslada los educativo y lo proyectamos en videojuegos, con el fin de que las clases sean más llamativa, motivadora, divertida y estimuladora al momento de trabajar en las aulas sea de manera virtual o presencial, de tal forma que se involucraran los alumnos de acuerdo a los componentes de la gamificación, de tal forma podríamos despertar su compromiso con las tareas asignadas, a la vez siento activos y entusiastas con las tareas trabajas.

Diseñar una estrategia metodológica para la enseñanza - aprendizaje de emprendimiento y gestión mediante la gamificación con el fin de aumentar la atención y concentración de acuerdo a los temas aprendidos. Por lo tanto, si nos concentramos en los conocimientos de los nuevos docentes referente al uso de las metodologías activas e innovadoras en los salones de clases, los estudiantes participan activamente con las nuevas metodologías aplicadas en la enseñanza.

Mediante el aprendizaje cooperativo podemos desarrollar la enseñanza-aprendizaje sea en las aulas o de manera presencial, ya que no poseemos de muchos conocimientos de los recursos didácticos virtuales, por lo que nos afecta al momento de transportar el aprendizaje a las clases virtuales, por lo que podremos basarnos para trabajar con el aprendizaje cooperativo y significativo para obtener el resultado poder resolver y la vez realizar de una manera didáctica para los estudiantes, teniendo muchos beneficios como pueden ser los siguientes:
a. El liderazgo
b. La afectividad
c. El respeto 
Revista Arbitrada Interdisciplinaria KOINONIA

Año VI. Vol VI. N4. Edición Especial: Educación III. 2021

Hecho el depósito de Ley: FA2016000010 ISSN: 2542-3088

FUNDACIÓN KOINONIA (F.K). Santa Ana de Coro. Venezuela.

Verónica Marisol Tene-Seas; Sandra Elizabeth Mena-Clerque
d. La solidaridad
e. Trabajo en equipo
f. La empatía

Además, incrementamos y a la vez motivamos a nuestros estudiantes para que posean nuevas habilidades, destrezas y conocimientos referente a la asignatura a trabajar. Emplear frecuentemente el uso de la gamificación en la clase para poder incrementar la motivación en el aprendizaje, de esa manera poder conseguir excelentes resultados académicos. Podemos también trabajar con videos y clase invertida, de acuerdo al tema a trabajar, de esa manera motivarlos e incentivarlos con los nuevos conocimientos que van adquirir por medio de los videos, al finalizar podríamos realizar un test para medir el nivel de los conocimientos adquiridos.

La gamificación debemos utilizarlo a diario en nuestras clases sea de manera virtual o presencial, ya que es una técnica que es de gran ayuda para poder motivar a los alumnos, con el fin de incrementar su participación activa en las clases tanto de manera individual o grupal, poniéndoles retos para que ellos mejoren su competitividad, pero de una manera sana y organizada sea con su grupo o individual, de esa manera seria una experiencia productiva y se realizara una clase dinámica tanto para los alumnos como para el docente.

Establecer que elementos de la gamificación podrían ser empleados para poder cumplir con los objetivos planteados y de esa manera aumentar el trabajo en equipo, el compañerismo, la igualdad y que aprendan aceptar sus errores, seria mediante los siguientes elementos:

a. Determinar las reglas del juego.

b. Concretar el contenido y características que sean coherentes para los estudiantes.

c. Especificar el propósito del juego desde un inicio.

d. Enunciar las regalas de forma clara, precisa y concisa. 
Revista Arbitrada Interdisciplinaria KOINONIA

Año VI. Vol VI. N4. Edición Especial: Educación III. 2021

Hecho el depósito de Ley: FA2016000010 ISSN: 2542-3088

FUNDACIÓN KOINONIA (F.K). Santa Ana de Coro. Venezuela.

Verónica Marisol Tene-Seas; Sandra Elizabeth Mena-Clerque

e. Crear grupos de forma equitativa.

f. Establecer la recompensa que obtendrán conforme vayan avanzando y al finalizar el juego.

Por consiguiente, se utilizarán los diferentes mecanismos que se plantearan desde un inicio de cualquier actividad, dejando en claro las dinámicas, mecánicas y componentes de las actividades a trabajar, ya que los componentes trabajan a la par con los elementos de la gamificación, teniendo como finalidad que ellos adquieran nuevos conocimientos y de esa manera un mejor desenvolvimiento.

\section{CONCLUSIONES}

A los alumnos les gustaría trabajar con los diferentes elementos de la gamificación, ya que en cada elemento se podría utilizar distintas formas para poder incentivar y de tal manera realizar las actividades que se tengan planificadas.

Que debemos tener muy en cuenta las herramientas con las que podemos trabajar ya que se concentran los conocimientos para aprender mediante juegos, por lo cual permite demostrar las diferentes habilidades y destrezas sea de una manera individual o grupal, siendo una forma de aprendizaje diferente y motivadora para cada uno de ellos.

Los alumnos consideran que en esta actualidad se debería trabajar con la gamificación de una manera permanente ya que es de gran ayudara para incentivarlos, motivarlos y tener una mejor atención y concentración de acuerdo a los conocimientos que se va a aprender, de esa manera podemos evidenciar como la gamificación contribuye en la educación obteniendo los mejores resultados académicos.

Se considera que se debería trabajar de una manera permanente con la utilización de la gamificación tanto en el aula como de manera virtual con el propósito de obtener buenos resultados académicos. 
Revista Arbitrada Interdisciplinaria KOINONIA

Año VI. Vol VI. N4. Edición Especial: Educación III. 2021

Hecho el depósito de Ley: FA2016000010 ISSN: 2542-3088

FUNDACIÓN KOINONIA (F.K). Santa Ana de Coro. Venezuela.

Verónica Marisol Tene-Seas; Sandra Elizabeth Mena-Clerque

\section{FINANCIAMIENTO}

No monetario.

\section{AGRADECIMIENTO}

A la Universidad Católica de Cuenca; por todo el apoyo brindado en la motivación y desarrollo de esta investigación.

\section{REFERENCIAS CONSULTADAS}

Bernal-Suarez, D., Martínez Pineda, M., Parra Pineda, A., \& Jiménez Hurtado, J. (2015). Investigación documental sobre calidad de la educación en instituciones educativas del contexto iberoamericano [Documentary Research on the Quality of Education in Educational Institutions of the Ibero-American Context]. Entramados: educación y sociedad, O(2), 107-124. Recuperado de http://fh.mdp.edu.ar/revistas/index.php/entramados/article/view/1389/1386

Burgos-Bonozo, M. F. (2019). Temas contables y la enseñanza aprendizaje de la asignatura de empredimiento y gestión [Accounting issues and teaching learning of the subject of entrepreneurship and management]. Ayan, 8(5), 55. http://repositorio.ug.edu.ec/handle/redug/39774

Carhuancho, I., Nolazco, F., Sicheri, L., Guerrero, M., \& Casana, K. (2019). Metodología para la investigación holística [Methodology for holistic research]. Recuperado de https://n9.cl/t0s2

Erazo-Álvarez, J. C. (2021). Capital intelectual y gestión de innovación: Pequeñas y medianas empresas de cuero y calzado en Tungurahua-Ecuador [Intellectual capital and innovation management: Small and medium-sized leather and footwear companies in Tungurahua - Ecuador]. Revista De Ciencias Sociales, 27, 230-245. Recuperado a partir de https://produccioncientificaluz.org/index.php/rcs/article/view/37004

Francés-Monllor, J. (2019). Análisis y aplicación de nuevas metodologías docentes basadas en clase invertida y gamificación a través de Moodle [Analysis and application of new teaching methodologies based on flipped class and gamification through Moodle]. Investigación e Innovación En La Enseñanza Superior. Nuevos Contextos, Nuevas Ideas, 527-540. http://hdl.handle.net/10045/98957 
Revista Arbitrada Interdisciplinaria KOINONIA

Año VI. Vol VI. N4. Edición Especial: Educación III. 2021

Hecho el depósito de Ley: FA2016000010

ISSN: 2542-3088

FUNDACIÓN KOINONIA (F.K). Santa Ana de Coro. Venezuela.

Verónica Marisol Tene-Seas; Sandra Elizabeth Mena-Clerque

García Casaus, F., Cara Muñoz, J. F., Martínez Sánchez, J. A., \& Cara Muñoz, M. M. (2021). La gamificación en el aula como herramienta motivadora en el proceso enseñanza-aprendizaje [Gamification in the classroom as a motivating tool in the teaching-learning process]. Logía, Educación Física y Deporte: Revista Digital de Investigación En Ciencias de La Actividad Física y Del Deporte, 1(2), 43-52. https://n9.cl/y2ic6

García, F., Cara, J., Martínez, J., \& Cara, M. (2020). La gamificación en el proceso de enseñanza-aprendizaje : una aproximación teórica [Gamification in the teachinglearning process: a theoretical approach]. Logía: Educación Física y Deporte, 1(1), $16-24$.

Gaspar, B., Reyes, M., González, F., \& García, F. (2016). La gamificacion y el aula invertida, aplicada en las universidades tecnológicas [Gamification and the flipped classroom, applied in technological universities]. Revista de Docencia e Investigación Educativa, 2(4), 30-33.

Liberio-Ambuisaca, X. P. (2019). El uso de las técnicas de gamificación en el aula para desarrollar las habilidades cognitivas de los niños y niñas de 4 a 5 años de educación inicial [The use of gamification techniques in the classroom to develop the cognitive skills of children from 4 to 5 years of Initial Education]. Conrado, 15(70), 392-397.

Méndez-Escobar, A. (2020). Educación en tiempos de pandemia (covid-19) [Education in times of pandemic (covid-19)]. Revista Universidad de La Salle, 1(85), 51-59. https://doi.org/10.19052/ruls.vol1.iss85.4

Ortiz-Colón, A. M., Jordán, J., \& Agredal, M. (2018). Gamificación en educación: una panorámica sobre el estado de la cuestión [Gamification in education: an overview of the state of the art]. Educação e Pesquisa, 44(0), 1-17. https://doi.org/10.1590/S1678-4634201844173773

Ricoy-Lorenzo, C. (2006). Contribución sobre los paradigmas de investigación [Contribution on research paradigms]. Educação, 31(1),11-22.

Torres-Toukoumidis, A., Romero-Rodríguez, L. M., Pérez-Rodríguez, M. A., \& Björk, S. (2018). Modelo teórico integrado de gamificación en ambientes E-learning (EMIGA) [Integrated theoretical model of gamification in E-learning environments (EMIGA)]. Revista Complutense de Educacion, 29(1), 129-145. https://doi.org/10.5209/RCED.52117 
Revista Arbitrada Interdisciplinaria KOINONIA

Año VI. Vol VI. N4. Edición Especial: Educación III. 2021

Hecho el depósito de Ley: FA2016000010 ISSN: 2542-3088

FUNDACIÓN KOINONIA (F.K). Santa Ana de Coro. Venezuela.

Verónica Marisol Tene-Seas; Sandra Elizabeth Mena-Clerque

Yánez-Analuisa, A. V. (2020). Estrategia didáctica para el aprendizaje de la asignatura de empredimiento y Gestion mediante el uso de las TIC [Didactic strategy for learning the Entrepreneurship and Management subject through the use of ICT]. Sustainability (Switzerland), http://repositorio.uisrael.edu.ec/handle/47000/2685

$4(1)$,

$1-9$.

(C2021 por los autores. Este artículo es de acceso abierto y distribuido según los términos y condiciones de la licencia Creative Commons Atribución-NoComercial-Compartirlgual 4.0 Internacional (CC BY-NC-SA 4.0) (https://creativecommons.org/licenses/by-nc-sa/4.0/). 